\title{
The effect of anterior lumbar interbody fusion staging order on perioperative complications in circumferential lumbar fusions performed within the same hospital admission
}

\author{
*Michael M. Safaee, MD, ${ }^{1}$ Alexander Tenorio, BA, ${ }^{1}$ Joseph A. Osorio, MD, PhD, ${ }^{1}$ \\ Winward Choy, MD, ${ }^{1}$ Dominic Amara, BA, ${ }^{1}$ Lillian Lai, BS, ${ }^{1}$ Serena S. Hu, MD, ${ }^{2}$ Bobby Tay, MD, ${ }^{3}$ \\ Shane Burch, MD, ${ }^{3}$ Sigurd H. Berven, MD, ${ }^{3}$ Vedat Deviren, MD, ${ }^{3}$ Sanjay S. Dhall, MD, ${ }^{1}$ \\ Dean Chou, MD, ${ }^{1}$ Praveen V. Mummaneni, MD, ${ }^{1}$ Charles M. Eichler, MD, ${ }^{4}$ Christopher P. Ames, MD, ${ }^{1}$ \\ and Aaron J. Clark, MD, PhD'
}

Departments of ${ }^{1}$ Neurological Surgery, ${ }^{3}$ Orthopedic Surgery, and ${ }^{4}$ Vascular Surgery, University of California, San Francisco; and ${ }^{2}$ Department of Orthopedic Surgery, Stanford University, Palo Alto, California

\begin{abstract}
OBJECTIVE Anterior lumbar interbody fusion (ALIF) is a powerful technique that provides wide access to the disc space and allows for large lordotic grafts. When used with posterior spinal fusion (PSF), the procedures are often staged within the same hospital admission. There are limited data on the perioperative risk profile of ALIF-first versus PSF-first circumferential fusions performed within the same hospital admission. In an effort to understand whether these procedures are associated with different perioperative complication profiles, the authors performed a retrospective review of their institutional experience in adult patients who had undergone circumferential lumbar fusions.

METHODS The electronic medicals records of patients who had undergone ALIF and PSF on separate days within the same hospital admission at a single academic center were retrospectively analyzed. Patients carrying a diagnosis of tumor, infection, or traumatic fracture were excluded. Demographics, surgical characteristics, and perioperative complications were collected and assessed.

RESULTS A total of 373 patients, 217 of them women (58.2\%), met the inclusion criteria. The mean age of the study cohort was 60 years. Surgical indications were as follows: degenerative disease or spondylolisthesis, 171 (45.8\%); adult deformity, 168 (45.0\%); and pseudarthrosis, 34 (9.1\%). The majority of patients underwent ALIF first (321 [86.1\%]) with a mean time of 2.5 days between stages. The mean number of levels fused was 2.1 for ALIF and 6.8 for PSF. In a comparison of ALIF-first to PSF-first cases, there were no major differences in demographics or surgical characteristics. Rates of intraoperative complications including venous injury were not significantly different between the two groups. The rates of postoperative ileus (11.8\% vs $5.8 \%, p=0.194)$ and ALIF-related wound complications $(9.0 \%$ vs $3.8 \%, p=0.283)$ were slightly higher in the ALIF-first group, although the differences did not reach statistical significance. Rates of other perioperative complications were no different.
\end{abstract}

CONCLUSIONS In patients undergoing staged circumferential fusion with ALIF and PSF, there was no statistically significant difference in the rate of perioperative complications when comparing ALIF-first to PSF-first surgeries.

https://thejns.org/doi/abs/10.3171/2020.6.FOCUS20296

KEYWORDS lumbar fusion; anterior approach; circumferential fusion; complications

$\mathrm{C}$ OMBINED anterior-posterior approaches to the lumbar spine play an important role in the treatment of degenerative disease, deformity, infection, tumor, and traumatic pathologies. ${ }^{1,2}$ These procedures can be performed as a single operation within one hospitalization, as two stages within a single hospitalization, or as two stages across separate hospitalizations. Staging depends on a variety of factors including the surgical indication, patient comorbidities, and access surgeon availability. For patients requiring circumferential fusion, there are no consensus

ABBREVIATIONS ALIF = anterior lumbar interbody fusion; $\mathrm{ASA}=$ American Society of Anesthesiologists; $\mathrm{CCl}=\mathrm{Charlson}$ Comorbidity Index; EBL = estimated blood loss; PSF = posterior spinal fusion; UTI = urinary tract infection

SUBMITTED April 5, 2020. ACCEPTED June 10, 2020.

INCLUDE WHEN CITING DOI: 10.3171/2020.6.FOCUS20296.

${ }^{*}$ M.M.S. and A.T. contributed equally to this work. 
guidelines on whether an anterior lumbar interbody fusion (ALIF) should be performed before or after a posterior spinal fusion (PSF).

Few studies have investigated the effect of staging anterior-posterior approaches on perioperative complications and clinical outcomes. In an effort to understand whether ALIF-first or PSF-first procedures were associated with different perioperative complication profiles, we performed a retrospective review of our institutional experience in adult patients who had undergone circumferential lumbar fusions. Perioperative complications were classified as either intraoperative or postoperative; intraoperative events included vascular injury, bowel perforation, or medical/cardiac event, whereas postoperative complications included anterior-related wound complications, hematoma, ileus, neurological deficit, or medical complications.

\section{Methods}

\section{Patient Identification}

Patients were identified through a retrospective search of all ALIF cases performed from 2007 to 2016 at the University of California, San Francisco. All activities were approved by our institutional review board. Adult patients (age $\geq 18$ years) who had undergone a PSF during the same admission were included in our analysis. Patients with an underlying diagnosis of tumor, infection, or trauma were excluded. Patients who had undergone ALIF-PSF on the same day were also excluded. All abdominal retroperitoneal exposures were performed by a fellowshiptrained vascular surgeon.

\section{Data Collection}

Patient demographics, surgical characteristics, and postoperative data were collected through a review of electronic medical records. Demographic data included age, sex, American Society of Anesthesiologists (ASA) classification, Charlson Comorbidity Index (CCI), BMI, and smoking status. Surgical indications were classified as follows: degenerative disease or spondylolisthesis, deformity, and pseudarthrosis. Surgical characteristics included order of anterior and posterior approaches, number of levels fused, estimated blood loss (EBL), and procedure duration. Surgical complications associated with the ALIF portion of the case were classified as intraoperative or postoperative. Intraoperative complications included vascular injury, bowel injury, or medical/cardiac event. Postoperative complications included ileus, anterior abdominal hematoma requiring surgical evacuation, anterior-related wound complications (dehiscence, infection, seroma, or abdominal hernia), or medical complications including cardiac event (new arrhythmia, congestive heart failure, or myocardial infarction), pulmonary event (pneumonia, pneumothorax, pulmonary edema, pleural effusion, chronic obstructive pulmonary disease exacerbation, or respiratory failure requiring reintubation), renal event (acute kidney injury or renal failure), urinary tract infection (UTI), and deep venous thrombosis or pulmonary embolus. Surgeries were classified as "ALIF first" if the ALIF had been performed before the posterior fusion on a separate day during the same hospitalization. Surgeries were classified as "PSF first" if the posterior fusion had been performed before the ALIF on a separate day during the same hospitalization.

\section{Statistical Analysis}

Univariate analysis was performed using the chi-square test or Fisher's exact test for categorical variables and the independent samples t-test for continuous variables. Statistical significance was defined as $p<0.05$. A multivariate model was generated using binary logistic regression with backward elimination. Variables included in the models were selected based on a significance of $p<0.100$ on univariate analysis. All analyses were performed using SPSS version 25 (IBM Corp.).

\section{Results}

\section{Patient Demographics}

A total of 373 patients, 217 of them women (58.2\%), underwent an ALIF and PSF on separate days during the same admission with a surgical indication of degenerative/ spondylolisthesis, deformity, or pseudarthrosis. The mean patient age was 60 years. The mean BMI was $29.0 \mathrm{~kg} / \mathrm{m}^{2}$ for the 371 patients with available data. ASA classification was available for 317 patients, with a distribution as follows: 9 class I (2.8\%), 200 class II (63.1\%), 106 class III (33.4\%), and 2 class IV (0.6\%) patients. The CCI scores were calculated for all patients, with a score of 0 in 227 $(60.9 \%)$ and a score of $1-2$ in $120(32.2 \%)$. There were 54 smokers (14.5\%) and 230 patients (61.7\%) with previous spine surgery. The mean follow-up was 25 months (range 0-115 months). Demographic data are summarized in Table 1.

\section{Surgical Characteristics}

Surgical indications were as follows: degenerative disease or spondylolisthesis, 171 (45.8\%); deformity, 168 (45.0\%); and pseudarthrosis, $34(9.1 \%)$. The majority of patients underwent ALIF first (321 [86.1\%]) compared to $52(13.9 \%)$ who underwent PSF first. The mean time between stages was 2.5 days (median 2.0 days). The overwhelming majority of patients (368 [98.7\%]) had a stage duration of 7 days or less. The remaining 5 patients were delayed because of cardiac events in 2 cases, severe ileus in 1 case, acute kidney injury in 1 case, and a planned stage duration of 8 days in 1 case. The mean number of levels fused by ALIF was 2.1 with 84 single-level fusions $(22.5 \%), 179$ two-level fusions $(48.0 \%), 97$ three-level fusions (26.0\%), and 13 four-level fusions (3.5\%). The mean EBL during ALIF was $269 \mathrm{ml}$ (range 30-3200 ml), and the mean ALIF procedure duration was 161 minutes (range 58-539 minutes). The mean number of levels fused by the posterior approach was 6.8 (range 1-18 levels fused) with a mean EBL of $1456 \mathrm{ml}$ (range $25-7500 \mathrm{ml}$ ) and a procedure duration of 277 minutes (range 52-714 minutes). Surgical characteristics are summarized in Table 2.

\section{Effect of ALIF Order on Staged Surgeries}

We performed a comparison of ALIF-first to PSF-first 
TABLE 1. Summary of characteristics among 373 patients who underwent ALIF

\begin{tabular}{cc}
\hline \multicolumn{1}{c}{ Variable } & Value \\
\hline Mean age in yrs & $60 \pm 12$ \\
\hline Female sex & $217(58.2)$ \\
\hline Mean BMl in $\mathrm{kg} / \mathrm{m}^{2 *}$ & $29.0 \pm 5.7$ \\
\hline ASA class $\dagger$ & $9(2.8)$ \\
\hline I & $200(63.1)$ \\
\hline II & $106(33.4)$ \\
\hline III & $2(0.6)$ \\
\hline IV & \\
\hline 0 & $227(60.9)$ \\
\hline 1 & $89(23.9)$ \\
\hline 2 & $31(8.3)$ \\
\hline 3 & $7(1.9)$ \\
\hline 4 & $9(2.4)$ \\
\hline 5 & $4(1.1)$ \\
\hline 6 & $5(1.3)$ \\
\hline 7 & $0(0)$ \\
\hline 8 & $1(0.3)$ \\
\hline Smoker & $54(14.5)$ \\
\hline Previous spine surgery & $230(61.7)$ \\
\hline Mean FU in mos (range) & $25(0-115)$ \\
\hline
\end{tabular}

$\mathrm{FU}=$ follow-up

Values are reported as the mean \pm standard deviation or number of patients (\%), unless indicated otherwise.

*Among 371 patients.

† Among 317 patients.

cases. There was no significant difference in age between the PSF-first and ALIF-first groups (61 vs 60 years, respectively, $\mathrm{p}=0.871)$, female sex $(59.6 \%$ vs $57.9 \%, \mathrm{p}=$ $0.821)$, or BMI (28.6 vs $\left.29.0 \mathrm{~kg} / \mathrm{m}^{2}, \mathrm{p}=0.663\right)$. ASA classification, CCI scores, surgical indications, and rates of smoking were not significantly different. The mean number of fused levels was slightly higher in the ALIF-first group (2.1 vs $1.9, \mathrm{p}=0.047$ ). The ALIF procedure duration and EBL were similar between groups. The mean levels fused by PSF (7.2 vs 6.8, respectively, $\mathrm{p}=0.534$ ) and PSF EBL (1736 vs $1406 \mathrm{ml}, \mathrm{p}=0.151$ ) were similar between the PSF- and ALIF-first groups; however, PSF duration was higher for the PSF-first group (338 vs 267 minutes, $\mathrm{p}<0.001$ ).

Perioperative complications were defined as intraoperative or postoperative. Intraoperative complications included injury to a major artery or vein, the bowel, or the urethra or a major medical/cardiac event. Rates of intraoperative venous injury were similar between ALIF- and PSF-first cases (3.4\% vs 5.8\%, respectively, $\mathrm{p}=0.425$ ). Postoperative complications included ileus, hematoma, ALIF-related wound complications (dehiscence, infection, or hernia), neurological deficit, or medical event. There was no difference in the overall postoperative complication rate (PSF: $32.7 \%$ vs $37.1 \%, p=0.543$ ). Rates of ileus
TABLE 2. Surgical characteristics among 373 patients who underwent ALIF

\begin{tabular}{|c|c|}
\hline Characteristic & Value \\
\hline \multicolumn{2}{|l|}{ Surgical indication } \\
\hline Degenerative/spondylolisthesis & $171(45.8)$ \\
\hline Deformity & $168(45.0)$ \\
\hline Pseudarthrosis & $34(9.1)$ \\
\hline \multicolumn{2}{|l|}{ Order of stages } \\
\hline Ant-pst & $321(86.1)$ \\
\hline Pst-ant & $52(13.9)$ \\
\hline Mean no. of days btwn stages (range) & $2.5(1-15)$ \\
\hline \multicolumn{2}{|l|}{ ALIF upper level } \\
\hline L2 & $18(4.8)$ \\
\hline L3 & $106(28.4)$ \\
\hline L4 & $178(47.7)$ \\
\hline L5 & $71(19.0)$ \\
\hline Mean no. of levels fused by ALIF (range) & $2.1(1-4)$ \\
\hline \multicolumn{2}{|l|}{ No. of levels fused by ALIF } \\
\hline 1 & $84(22.5)$ \\
\hline 2 & $179(48.0)$ \\
\hline 3 & $97(26.0)$ \\
\hline 4 & $13(3.5)$ \\
\hline ALIF procedure time in mins (range) & $161(58-539)$ \\
\hline ALIF EBL in $\mathrm{ml}$ (range) ${ }^{*}$ & $269(30-3200)$ \\
\hline Mean no. of levels fused by PSF (range) & $6.8(1-18)$ \\
\hline PSF procedure time in mins (range) & $277(52-714)$ \\
\hline PSF EBL in ml (range) $\dagger$ & $1456(25-7500)$ \\
\hline Length of stay in days (range) & $9.5(3-46)$ \\
\hline
\end{tabular}

$(11.8 \%$ vs $5.8 \%, p=0.194)$ and ALIF-related wound complications $(9.0 \%$ vs $3.8 \%, p=0.283)$ were slightly higher for the ALIF-first group. Rates of other postoperative complications were not significantly different. Length of stay and long-term follow-up were also not significantly different between the two groups. These results are summarized in Table 3.

\section{Multivariate Analysis}

Two multivariate models were generated to identify potential factors associated with intraoperative and postoperative complications. Variables were selected based on a significance of $p<0.100$ on univariate analysis. Although not significant in the univariate analysis, ALIF-first was also included in the multivariate models. The model for intraoperative complications included age, ALIF EBL, ALIF duration, ASA class, and ALIF first. In this model, only ALIF EBL was associated with the presence of an intraoperative complication. The model for postoperative complications included age, BMI, ALIF procedure duration, PSF procedure duration, PSF EBL, surgical 
TABLE 3. Effect of ALIF order on staged procedures

\begin{tabular}{|c|c|c|c|}
\hline Variable & $\begin{array}{l}\text { PSF-First } \\
\text { Group }\end{array}$ & $\begin{array}{l}\text { ALIF-First } \\
\text { Group }\end{array}$ & $\begin{array}{c}p \\
\text { Value }\end{array}$ \\
\hline No. of patients & 52 & 321 & \\
\hline Age in yrs & 61 & 60 & 0.871 \\
\hline Female sex & $31(59.6)$ & $186(57.9)$ & 0.821 \\
\hline $\mathrm{BMl}$ in $\mathrm{kg} / \mathrm{m}^{2 *}$ & 28.6 & 29.0 & 0.663 \\
\hline \multicolumn{4}{|l|}{ ASA class* } \\
\hline I-II & $24(57.1)$ & $185(67.3)$ & 0.222 \\
\hline III-IV & $18(42.9)$ & $90(32.7)$ & 0.222 \\
\hline \multicolumn{4}{|l|}{$\mathrm{CCl}$ score } \\
\hline $0-2$ & $48(92.3)$ & $299(93.1)$ & $0.771 \dagger$ \\
\hline $3-5$ & $4(7.7)$ & $16(5.0)$ & $0.501 \dagger$ \\
\hline $6-8$ & $0(0)$ & $6(1.9)$ & $0.99 \dagger$ \\
\hline \multicolumn{4}{|l|}{ Surgical indication } \\
\hline Degenerative/spondylolisthesis & $19(36.5)$ & $152(47.4)$ & 0.147 \\
\hline Deformity & $27(51.9)$ & $141(43.9)$ & 0.282 \\
\hline Pseudarthrosis & $6(11.5)$ & $28(8.7)$ & 0.513 \\
\hline Smoker & $8(15.4)$ & $46(14.3)$ & 0.841 \\
\hline Mean no. of ALIF levels fused & 1.9 & 2.1 & 0.047 \\
\hline ALIF procedure time in mins & 183 & 157 & 0.065 \\
\hline ALIF EBL in $\mathrm{ml}^{*}$ & 317 & 261 & 0.408 \\
\hline Mean no. of PSF levels fused & 7.2 & 6.8 & 0.534 \\
\hline PSF procedure time in mins & 338 & 267 & $<0.001$ \\
\hline PSF EBL in $\mathrm{ml}^{*}$ & 1736 & 1406 & 0.151 \\
\hline Intraop complications, any & $3(5.8)$ & $12(3.7)$ & $0.449 \dagger$ \\
\hline Arterial injury & $0(0)$ & $0(0)$ & - \\
\hline Venous injury & $3(5.8)$ & $11(3.4)$ & $0.425 \dagger$ \\
\hline Bowel/ureteral injury & $0(0)$ & $0(0)$ & - \\
\hline Medical/cardiac event & $0(0)$ & $1(0.3)$ & $0.99 \dagger$ \\
\hline Postop complications, any & $17(32.7)$ & $119(37.1)$ & 0.543 \\
\hline lleus & $3(5.8)$ & $38(11.8)$ & 0.194 \\
\hline Hematoma & $0(0)$ & $3(0.9)$ & $0.99 \dagger$ \\
\hline Ant wound complication & $2(3.8)$ & $29(9.0)$ & $0.283 \dagger$ \\
\hline Neurological deficit & $0(0)$ & $1(0.3)$ & $0.99 \dagger$ \\
\hline Medical event, any & $13(25.0)$ & $76(23.7)$ & 0.835 \\
\hline Cardiac & $4(7.7)$ & $23(7.2)$ & $0.779 \dagger$ \\
\hline Pulmonary & $3(5.8)$ & $26(8.1)$ & $0.781 \dagger$ \\
\hline Renal & $0(0)$ & $8(2.5)$ & $0.606 \dagger$ \\
\hline UTI & $3(5.8)$ & $17(5.3)$ & $0.749 \dagger$ \\
\hline DVT/PE & $1(1.9)$ & $4(1.2)$ & $0.530 \dagger$ \\
\hline Length of stay in days & 11.1 & 9.3 & 0.075 \\
\hline FU in mos & 29.9 & 24.6 & 0.155 \\
\hline
\end{tabular}

DVT = deep venous thrombosis; $\mathrm{PE}=$ pulmonary embolus.

Values expressed as number (\%), unless indicated otherwise. Boldface type indicates statistical significance.

${ }^{*}$ Among patients with complete data.

$\dagger$ Fisher's exact test.
TABLE 4. Multivariate analysis of variables associated with postoperative complications

\begin{tabular}{lcc}
\hline Variable & OR $(95 \% \mathrm{Cl})$ & p Value \\
\hline Age & $1.039(1.018-1.061)$ & $<0.001$ \\
\hline BMI & $1.041(1.001-1.083)$ & $\mathbf{0 . 0 4 7}$ \\
\hline PSF duration & $1.002(1.000-1.004)$ & $\mathbf{0 . 0 2 7}$ \\
\hline
\end{tabular}

Boldface type indicates statistical significance.

indication, and ALIF first. In this model, age (OR 1.039, 95\% CI 1.018-1.061, p < 0.001), BMI (OR 1.041, 95\% CI $1.001-1.083, \mathrm{p}=0.047)$, and PSF duration (OR 1.002, 95\% CI $1.000-1.004, \mathrm{p}=0.027$ ) were associated with a postoperative complication. These results are summarized in Table 4.

\section{Discussion}

ALIF is a versatile surgical technique with roles in the treatment of degenerative disease, deformity, tumor, infection, and pseudarthrosis. There are no consensus guidelines on the role of staging ALIF-PSF procedures, and data on the perioperative complications related to ALIF-first or PSF-first surgery are limited. In this study, we attempted to identify associations between the order of ALIF and ALIF-related perioperative complications.

The major notable finding of this study was that the ALIF order did not affect perioperative ALIF-related complications. The rates of intraoperative complications (arterial injury, venous injury, bowel injury, ureteral injury, and medical event) and postoperative complications (ileus, hematoma, wound complications, neurological deficit, and medical) were not significantly different between the PSF-first and ALIF-first cohorts. Therefore, ALIF order is unlikely to have a major impact on the perioperative risk profile for patients undergoing circumferential fusion and should not be a major factor in the decision-making process for staging the procedures.

There are limited data investigating the role of ALIFfirst compared to PSF-first procedures in circumferential fusion. In a review of the National Inpatient Sample (NIS) from 1998 to 2006, Memtsoudis et al. found that patients undergoing anterior-posterior fusion were younger than those undergoing posterior approaches and had longer stays, higher procedure-related complications, higher rates of blood transfusions, and higher mortality, although the latter was primarily related to patients with metastatic disease and trauma. ${ }^{3}$ The highest rates of fatal outcomes and complications were associated with an anterior thoracic approach, consistent with other reports in the literature, ${ }^{4}$ however, the ALIF carries a significantly lower risk profile than the transthoracic approach. ${ }^{5,6}$ Hassanzadeh et al. reviewed 63 patients undergoing combined anteriorposterior fusion for adult deformity and compared those with surgeries staged less than 21 days apart to those with procedures greater than 21 days apart? ${ }^{7}$ There were no statistically significant differences in total EBL, major complications, or total hospital stay; however, a longer staging time was associated with fewer blood transfusions. These 
authors did not assess differences in anterior-first versus posterior-first surgical plans.

Tsirikos et al. analyzed a cohort of 45 pediatric patients undergoing anterior-posterior fusion and found increased intraoperative blood loss, operative time, and medical complications in the single-stage cohort. ${ }^{8}$ In adult deformity, Rhee et al. utilized a staged approach for high-risk revision cases by separating the posterior surgery into two smaller procedures in order to limit hemodynamic risk. ${ }^{9}$ Hassanzadeh et al. demonstrated that staging anterior and posterior procedures by 21 days resulted in reductions in combined EBL, perioperative complications, and total hospital stay; however, none of these factors met statistical significance. ${ }^{7}$ None of these studies specifically addressed the role of ALIF-first or PSF-first procedures with respect to blood loss and perioperative complication risk profile. We showed that among patients undergoing circumferential lumbar fusion during a single hospital admission, ALIF stage order is not associated with the presence of a perioperative complication. In a multivariate model assessing factors associated with an intraoperative complication, the only significant association was with ALIF EBL, which was expected given the blood loss associated with an intraoperative venous injury. No other variables were associated with the presence of an intraoperative complication. In a separate model assessing postoperative complications, only age, BMI, and PSF duration were associated with the presence of a postoperative complication. Age and procedure duration are well-recognized risk factors ${ }^{10-16}$ and our group has previously identified obesity as a risk factor for postoperative complications after ALIF, particularly with respect to ileus, wound-related complications, and UTI. ${ }^{17}$

Our typical institutional practice is to utilize an ALIFfirst approach, particularly for complex cases. There are several advantages to this approach such as maximizing graft size without the need to overpower posterior instrumentation. This allows the surgeon to assess post-ALIF lordosis and determine the need for additional posterior column osteotomies. In patients with radicular symptoms who do not improve with ALIF, additional foraminotomies can be performed during the posterior stage. In this series, a PSF-first approach was most commonly used in cases in which a planned transforaminal or lateral lumbar interbody fusion had been aborted, an ALIF had been recommended based on intraoperative findings after PSF, or there had been limited access surgeon availability. Given the design of this study, it was not possible to identify the reason for PSF-first approaches in each individual case; however, we do not believe such knowledge would have altered our results. There are several limitations to this study, most notably its retrospective design and primary focus on perioperative complications rather than longterm complications such as pseudarthrosis, proximal junctional failure, graft subsidence, or rod fracture.

\section{Conclusions}

Among patients undergoing circumferential lumbar fusion with ALIF and PSF, there was no significant difference in perioperative complications between those who had undergone ALIF-first procedures and those who had undergone PSF-first procedures. In a multivariate model, age, BMI, and PSF procedure duration were associated with the presence of a postoperative complication. In appropriately selected patients, the perioperative risk profile of ALIF does not change when performed before or after a PSF.

\section{Acknowledgments}

Dr. Tay has received an Institutional Fellowship Grant. Dr. Ames has received grant funding from the SRS.

\section{References}

1. Sundaresan N, Steinberger AA, Moore F, et al. Indications and results of combined anterior-posterior approaches for spine tumor surgery. J Neurosurg. 1996;85(3):438-446.

2. Wright N. Single-surgeon simultaneous versus staged anterior and posterior spinal reconstruction: a comparative study. J Spinal Disord Tech. 2005;18(suppl):S48-S57.

3. Memtsoudis SG, Vougioukas VI, Ma Y, et al. Perioperative morbidity and mortality after anterior, posterior, and anterior/posterior spine fusion surgery. Spine (Phila Pa 1976). 2011;36(22):1867-1877.

4. Shen Y, Silverstein JC, Roth S. In-hospital complications and mortality after elective spinal fusion surgery in the United States: a study of the nationwide inpatient sample from 2001 to 2005. J Neurosurg Anesthesiol. 2009;21(1):21-30.

5. Garg J, Woo K, Hirsch J, et al. Vascular complications of exposure for anterior lumbar interbody fusion. J Vasc Surg. 2010;51(4):946-950.

6. Mobbs RJ, Phan K, Daly D, et al. Approach-related complications of anterior lumbar interbody fusion: results of a combined spine and vascular surgical team. Global Spine J. 2016;6(2):147-154.

7. Hassanzadeh H, Gjolaj JP, El Dafrawy MH, et al. The timing of surgical staging has a significant impact on the complications and functional outcomes of adult spinal deformity surgery. Spine J. 2013;13(12):1717-1722.

8. Tsirikos AI, Chang WN, Dabney KW, Miller F. Comparison of one-stage versus two-stage anteroposterior spinal fusion in pediatric patients with cerebral palsy and neuromuscular scoliosis. Spine (Phila Pa 1976). 2003;28(12):1300-1305.

9. Rhee JM, Bridwell KH, Lenke LG, et al. Staged posterior surgery for severe adult spinal deformity. Spine (Phila Pa 1976). 2003;28(18):2116-2121.

10. Lau D, Osorio JA, Deviren V, Ames CP. The relationship of older age and perioperative outcomes following thoracolumbar three-column osteotomy for adult spinal deformity: an analysis of 300 consecutive cases. J Neurosurg Spine. 2018; 28(6):593-606.

11. Pellisé F, Vila-Casademunt A, Núñez-Pereira $S$, et al. The Adult Deformity Surgery Complexity Index (ADSCI): a valid tool to quantify the complexity of posterior adult spinal deformity surgery and predict postoperative complications. Spine J. 2018;18(2):216-225.

12. Passias PG, Horn SR, Jalai CM, et al. Comparative analysis of perioperative complications between a multicenter prospective cervical deformity database and the Nationwide Inpatient Sample database. Spine J. 2017;17(11):1633-1640.

13. Scheer JK, Smith JS, Schwab F, et al. Development of a preoperative predictive model for major complications following adult spinal deformity surgery. J Neurosurg Spine. 2017; 26(6):736-743.

14. Soroceanu A, Burton DC, Oren JH, et al. Medical complications after adult spinal deformity surgery: incidence, risk factors, and clinical impact. Spine (Phila Pa 1976). 2016;41(22): $1718-1723$. 
15. Smith JS, Klineberg E, Lafage V, et al. Prospective multicenter assessment of perioperative and minimum 2-year postoperative complication rates associated with adult spinal deformity surgery. J Neurosurg Spine. 2016;25(1):1-14.

16. Narain AS, Parrish JM, Jenkins NW, et al. Risk factors for medical and surgical complications after single-level minimally invasive transforaminal lumbar interbody fusion. Int $J$ Spine Surg. 2020;14(2):125-132.

17. Safaee MM, Tenorio A, Osorio JA, et al. The impact of obesity on perioperative complications in patients undergoing anterior lumbar interbody fusion. J Neurosurg Spine. 2020;33(3):332-341.

\section{Disclosures}

Dr. Tay is a consultant for Biomet, Synthes, Stryker, Lumetra, Omega, and NuVasive. Dr. Berven has ownership of Green Sun Medical and Providence Medical; is a consultant for Medtronic, Stryker, Innovasis, Globus, Medicrea, and Integrity; and receives royalties from Medtronic and Stryker. Dr. Deviren is a consultant for NuVasive, Atec/Alphatec, Biomet, Seaspine, and Medicrea and receives royalties from NuVasive. Dr. Dhall receives honoraria from DePuy and Globus. Dr. Chou is a consultant for and receives royalties from Globus. Dr. Mummaneni is a consultant for Stryker, Globus, and DePuy Synthes; has direct stock ownership in Spinicity/ISD; receives royalties from DePuy Synthes, Thieme Publishers, and Springer Publishers; and receives support from ISSG, NREF, and AO Spine for non-study-related clinical or research effort. Dr. Eichler is a consultant for Medtronic Spine and NuVasive. Dr. Ames receives royalties from Stryker, Biomet
Zimmer Spine, DePuy Synthes Spine, NuVasive, Next Orthosurgical, K2M, and Medicrea; is a consultant for DePuy Synthes, Medtronic, Medicrea, and K2M; conducts research for Titan Spine, DePuy Synthes, and ISSG; serves on the editorial board of Operative Neurosurgery; serves on the Executive Committee of ISSG; and is the Director of Global Spinal Analytics. Dr. Clark is a consultant for NuVasive and receives support from NuVasive for non-study-related clinical or research effort.

\section{Author Contributions}

Conception and design: Clark, Safaee, Tenorio, Ames. Acquisition of data: all authors. Analysis and interpretation of data: Clark, Safaee, Tenorio. Drafting the article: Clark, Safaee, Tenorio. Critically revising the article: Clark, Safaee, Tenorio. Reviewed submitted version of manuscript: Clark, Safaee, Tenorio, Osorio, $\mathrm{Hu}$, Tay, Burch, Berven, Deviren, Dhall, Chou, Mummaneni, Eichler, Ames. Approved the final version of the manuscript on behalf of all authors: Clark. Statistical analysis: Clark, Safaee, Tenorio. Administrative/technical/material support: Clark. Study supervision: Clark.

\section{Correspondence}

Aaron J. Clark: University of California, San Francisco, CA. aaron.clark@ucsf.edu. 